\section{Quo vadis ECMO?}

Die Geburtsstunde der extrakorporalen Zirkulation (EKZ) schlug 1952, als Gibbon erstmals klinisch mit einem derartigen System Operationen am offenen Herzen durchführte [1]. 1958 hat Zenker in Marburg die erste erfolgreiche Operation am offenen Herzen in Deutschland mit Hilfe einer HerzLungen-Maschine vorgenommen. Innerhalb weniger Jahre weitete sich das Indikationsspektrum auf die Behandlung von massiven Lungenembolien [2] und kardiogenen Schockzuständen nach Myokardinfarkt [3].

Heute stehen uns verschiedene Verfahren der extrakorporalen Zirkulation zur Verfügung, die sich im Wesentlichen in zwei Gruppen gliedern: 1. EKZ kurzzeitig im Rahmen einer Operation, 2. EKZ z. B. in Form der extrakorporalen Membranoxygenierung (ECMO) zum (auch) längerfristigen Ersatz bei Versagen von Lunge und/oder Herz.

Tosson u. Mitarb. beschreiben in der in diesem Heft publizierten Arbeit [4] die aktuellen Einsatzmöglichkeiten derartiger Verfahren zur Behandlung von pulmonalen Erkrankungen anhand von Fallbeispielen. So wird eine Patientin mit schwerer pulmonaler Hypertonie geschildert, die sich einer Herz-Lungen-Transplantation unterzog, die naturgemäß unter den Bedingungen der EKZ erfolgte. Isolierte Lungentransplantationen - ein- und sequentiell beidseitig - bedürfen der extrakorporalen Zirkulation nur relativ selten und zwar insbesondere bei Patienten mit einer pulmonalen Hypertonie als Grunderkrankung.

Daneben diskutieren die Autoren aber auch die ECMO und zwar sowohl für den kurzfristigen (schwere Lungenembolie, therapeutische Ganzlungenlavage bei Alveolarproteinose) als auch den längerfristigen (gastrobronchiale Fistel) Einsatz.

Die ECMO stellt eine Adaption der konventionellen kardiopulmonalen Bypasstechniken dar, die in den 70er Jahren in die Therapie des Adult Respiratory Distress Syndroms (ARDS) eingeführt wurde. Die dem Einsatz der ECMO für die Indikation ARDS zugrundeliegende Hypothese ist, dass Oxygenierung und $\mathrm{CO}_{2}$-Elimination durch ein extrakorporales System ermöglichen, die Lunge bis zur Wiederherstellung „ruhen“ zu lassen - Faktoren wie Volu- und Barotrauma sowie Sauerstofftoxizität spielen praktisch keine Rolle mehr. Nachdem die initialen Berichte enthusiastisch waren, wurden 1979 [5] und

Pneumologie 2001; 55: 404-405

(c) Georg Thieme Verlag Stuttgart · New York ISSN 0934-8387

\section{Vogelmeier}

Klinik für Innere Medizin, Schwerpunkt Pneumologie, Klinikum der Philipps-Universität, Marburg

1994 [6] kontrollierte klinische Studien publiziert, die beide keinen Vorteil für die ECMO-Therapie erbrachten. Die Überlebensraten in den ECMO-Gruppen betrugen nur 10 bzw. 33\%. Bei Neugeborenen mit akutem Lungenversagen (IRDS) hingegen ist das Verfahren zweifellos erfolgreich mit Überlebensraten von bis $\mathrm{zu} 80 \%$.

Seither wurde die ECMO-Technik weiterentwickelt. Das ECMO-Verfahren wurde sicherer durch heparinisierte Systeme und transkutan zu legende Zugänge. Mit diesen neuen Techniken berichten einige Zentren über hervorragende Ergebnisse mit Überlebensraten jenseits von $50 \%$ bei schwerer respiratorischer Insuffizienz [7-9]. Kontrollierte Studien unter Anwendung der verbesserten Verfahren fehlen aber bislang.

Im Gegensatz dazu konnten zwischenzeitlich mit auf die pathophysiologischen Veränderungen abgestimmten konventionellen Beatmungsstrategien eindeutige Überlebensvorteile erzielt werden. So wurde gezeigt, dass eine „lungenprotektive“ Beatmung mit niedrigen Beatmungsdrucken, geringen Tidalvolumina und positiv endexspiratorischen Drucken oberhalb des unteren Inflektionspunkts der Druck-Volumenkurve („open lung approach“) das Überleben von ARDS-Patienten signifikant verbessert [10-13]. Weitere Innovationen stellen die inhalative Therapie mit NO, die lokale Surfactantapplikation und die Flüssigkeitsventilation (partial liquid ventilation) dar. Die letztgenannten Verfahren sind allerdings bislang einen eindeutigen Effektivitätsnachweis schuldig geblieben. Zentren, die alle genannten Strategien nutzen können, machen die Erfahrung, dass sich bei optimaler Anwendung konventioneller Beatmung die Indikation zur ECMO nur noch selten stellt.

Zusammenfassend lässt sich festhalten, dass die ECMO auf der Basis der geschilderten Datenlage nach wie vor ein experimentelles Therapieverfahren darstellt. In ausgewählten Fällen kann die ECMO ohne Zweifel, wie auch die eindrucksvollen Kasuistiken von Tosson u. Mitarb. belegen, wesentliche Erfolge bringen. Um dies zu gewährleisten, sollte die ECMO aber nur in wenigen erfahrenen Zentren eingesetzt werden. Unsere Hauptanstrengungen für die Zukunft sollten darauf gerichtet sein, die der respiratorischen Insuffizienz eines individuellen Patienten zugrunde liegende Pathophysiologie besser $z u$ erfassen und unsere therapeutischen Maßnahmen daran anzupassen. 


\section{Literatur:}

${ }^{1}$ Gibbon JH. Application of a mechanical heart and lung apparatus to cardiac surgery. Minn Med 1954; 37: 171

${ }^{2}$ Cooley DA, Beall AC, Alexander JL. Acute massive pulmonary embolism: Successful surgical treatment using temporary cardiopulmonary bypass. JAMA 1961; 177: 283

${ }^{3}$ Stuckey JH, Newman MM, Dennis C et al. The use of the heartlung machine in selected cases of acute myocardial infarction. Surg Forum 1957; 8: 342

${ }^{4}$ Tosson R, Klak K, Sivitanidis E et al. Die extrakorporale Kreislaufzirkulation bei der Behandlung von Lungenkrankheiten. Pneumologie 2001; 55: 409-413

${ }^{5}$ Zapol R, Leasa D, Sanderson M et al. Extracorporeal membrane oxygenation in severe acute respiratory failure. JAMA 1979; 242: 2193

${ }^{6}$ Morris AH, Wallace CJ, Menlove RL et al. Randomized clinical trial of pressure-controlled inverse ratio ventilation and extracorporeal $\mathrm{CO} 2$ removal for adult respiratory distress syndrome. Am J Respir Crit Care Med 1994; 149: 838

${ }^{7}$ Lewandowski K. Extracorporeal membrane oxygenation for severe acute respiratory failure. Crit Care 2000; 43: 156

${ }^{8}$ Mols G, Loop T, Hermle $G$ et al. 10 years experience with extracorporeal membrane oxygenation. Anästhesiol Intensivmed Notfallmed Schmerzther 2001; 36: 4

${ }^{9}$ Linden V, Palmer K, Reinhard J et al. High survival in adult patients with acute respiratory distress syndrome treated by extracorporeal membrane oxygenation, minimal sedation, and pressure supported ventilation. Intensive Care Med 2000; 26 : 1630

10 Jürgens E, Roissant R. Invasive Beatmung beim hypoxämischen Lungenversagen - State of the Art. Intensivmed 2000; 37: 257

${ }^{11}$ Amato MB, Barbas CS, Medeiros DM et al. Effect of a protectiveventilation strategy on mortality in the acute respiratory distress syndrome. N Engl J Med 1998; 338: 347

12 The Acute Respiratory Distress Syndrome Network. Ventilation with lower tidal volumes as compared with traditional tidal volumes for acute lung injury and the acute respiratory distress syndrome. N Engl J Med 2000; 342: 1301

${ }^{13}$ Tobin MJ. Advances in mechanical ventilation. N Engl J Med 2001; 344: 1986

Prof. Dr. med. Claus Vogelmeier

Klinik für Innere Medizin, Schwerpunkt Pneumologie

Klinikum der Philipps-Universität Marburg

Baldingerstraße

35043 Marburg

\section{Emphysem-Hotline}

Wir haben in der LVA Klinik Bad Reichenhall eine sogenannte Emphysem-Hotline eingerichtet unter 01805-EMPHYSEM oder 01805/367497 (0,24 DM/Minute), wo Betroffene und Interessierte Fragen zum Thema „Emphysem“ stellen können. Diese Telefonhotline wird durch eine Mitarbeiterin betreut und kompetente Ärzte werden fachliche und medizinische Fragen beantworten. Außerdem möchten wir hilfreiche Tipps zu wichtigen Kontaktadressen wie Selbsthilfegruppen, Beratungsstellen u. v. m. weitergeben.

In diesem Zusammenhang bieten wir auch eine Internetseite zum Thema „Emphysem“ an: http://www.emphysem-info.de. Hier werden in leicht verständlicher Art Menschen, die an Lungenemphysem erkrankt sind, ihren Angehörigen und vielleicht auch Ärzten und Fachleuten Informationen über die Krankheit, ihre Entstehung und Behandlung, aber auch Nachrichten zu neuen Behandlungsmethoden, gesundheitspolitischen Meldungen oder aktuellen Forschungsergebnissen zugänglich gemacht. Ziel unserer Arbeit ist es, Hilfe und Unterstützung zu geben, damit Betroffene besser mit dieser chronischen Krankheit klarkommen.

Diese Webseite und unsere Emphysem-Hotline sollen sich gegenseitig ergänzen.

Man kann uns weiterhin erreichen:

Emphysem-Hotline an der Klinik Bad Reichenhall der LVA Niederbayern-Oberpfalz, z. Hd. Frau Böckel, Salzburger Straße 8-11, 83435 Bad Reichenhall, Tel. 01805/367497 oder 01805/EMPHYSEM, Fax: 08651/602745, E-mail: hotline@emphysem-info.de 